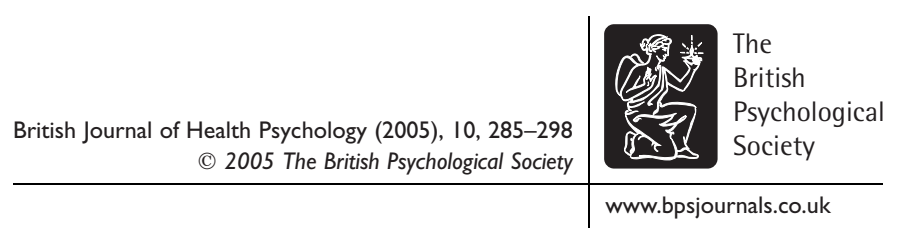

\title{
The DAS24: A short form of the Derriford Appearance Scale DAS59 to measure individual responses to living with problems of appearance
}

\author{
Tony Carr', Timothy Moss ${ }^{2} *$ and David Harris ${ }^{3}$ \\ 'Sub-Department of Clinical Psychology, University of Plymouth, UK \\ ${ }^{2}$ Centre for Appearance Research, University of the West of England, UK \\ ${ }^{3}$ Department of Reconstructive and Plastic Surgery, Derriford Hospital, Plymouth, \\ UK
}

\begin{abstract}
Objectives. To develop a psychometrically robust and widely applicable short form of the Derriford Appearance Scale, (DAS59), which (I) will reliably and validly assess the distress and difficulties experienced in living with problems of appearance, (2) is acceptable to clinical and non-clinical populations, and (3) facilitates research and clinical decision-making through good standardization and sensitivity.
\end{abstract}

Design. Cross-sectional survey designs using clinical (out-patient and in-patient) and general population samples.

Method. Twenty-five items were selected initially from the 59 items of the original DAS59. These were refined to 24 through item analyses and the scale was standardized on 535 patients with a range of problems of appearance and on a representative general population sample $(\mathrm{N}=\mathrm{I}, \mathrm{I07})$.

Results. All 24 items contributed well to the total score and internal consistency was high ( $\alpha=.92)$. Test-retest reliability (6 months) was good $(0.82)$, and criterion validity, with the DAS59, was excellent (0.88). Good construct validity was demonstrated in differences between (I) patient and general population samples, (2) members of the general population concerned and not concerned about their appearance, and (3) in patterns of convergent and divergent correlations with a range of established scales. The general population data revealed widespread concerns about appearance.

Conclusion. The DAS24 provides a widely applicable and acceptable short form of the original DAS59. It is psychometrically robust and discriminates well between patient groups, between clinical and non-clinical populations, and within the general population between those concerned, and those not concerned, about their appearance.

* Correspondence should be addressed to Dr Timothy Moss, Centre for Appearance Research, School of Psychology, University of the West of England, Coldharbour Lane, Bristol BSI6 IQY, UK (e-mail: tim.moss@uwe.ac.uk). 
It has previously been argued (Carr, 1997a, 1997b; Carr, Harris, \& James, 2000; Moss 1997a) that vital work to further develop our understanding of problems of appearance and to evolve more effective therapeutic strategies for those suffering from levels of self-consciousness of appearance that are clinically significant, bodyimage disturbance, and body dysmorphic disorder, has been severely impeded by the lack of appropriate measurement approaches. Measurement of the perceptual dimension of body-image (e.g. Slade \& Russell, 1973) marked the beginning of a process of development of measures of body-image that has reflected the increasing elaboration of the concept to include behavioural, affective, and cognitive dimensions. However, the majority of measures have not been psychometrically developed beyond their first description, and even where measures have been standardized, all too often this has been based on small samples of non-patient, student, caucasian, females (Cash \& Pruzinsky, 2002; Thompson, Heinberg, Altabe, \& Tantleff-Dunn, 1999).

Following an extensive review, Carr (2002) identified a small number of viable scales and structured interviews for assessing body-image disturbance, self-consciousness of appearance, and body dysmorphic disorder. These range from the Body-image Avoidance Questionnaire (BIAQ; Rosen, Srebnik, Saltzburg, \& Wendt, 1991) for assessing behavioural aspects of body-image disturbance, to the Appearance Schemas Inventory (ASI; Cash \& Labarge, 1996) for assessing cognitive aspects of body-image. The Body Dysmorphic Disorder Examination (BDDE; Rosen, Reiter, \& Orosan, 1995) is discussed as a well validated diagnostic interview, as is the Eating Disorders Examination (EDE; Cooper \& Fairburn, 1987) for an interview-based assessment of eating disorders and associated disturbances of body-image.

In developing the BIAQ (Rosen et al., 1991), the authors acknowledge the multidimensionality of body-image and the consequent need to assess related behaviours, in addition to the dimensions covered by existing alternative assessment methods. The BIAQ is a 19-item self-report scale based upon the weight and shape concerns of female undergraduates. There is some psychometric development, and overall, the psychometric properties are either adequate or good. However, the items reveal a strong representation of weight and eating concerns, resulting directly from the questions asked of the female undergraduates in generating the original item set. This emphasis upon weight and shape emerged in the factor analysis and in correlations with criterion measures. The BIAQ cannot be used with confidence to assess behavioural aspects of body-image disturbance that are unrelated to weight and shape or which occur most frequently in clinical populations.

From the basic premise of the central role of appearance schema in processing physical appearance information relevant to the self, Cash, and Labarge (1996) devised the ASI. The 14 items tap into beliefs about the importance, meaning, and perceived influences of appearance in a person's life. However, the item generation process is not specified, and there is no stage of item endorsement and no subsequent refinement of the item set. Detailed psychometric development was undertaken on 274 female college students, revealing only adequate internal consistency and criterion validities, and no information on temporal stability. Although clearly needing further psychometric development, standardization, and data on both males and a range of clinical samples, the ASI is already finding applications in research, for example, Grant and Cash (1995), and Labarge, Cash, and Brown, (1998). 
In addition to dimensional measures of body-image disturbance, there is a pressing need (from both clinical and research perspectives) for approaches that assess the totality of body-image disturbance. The standardized interview version of the BDDE (Rosen et al., 1995) is widely used but it lacks normative data. Also, the usual duration of 1.5 hours renders it impracticable in many settings. The self-report version of the BDDE (Rosen \& Ramirez, 1998) overcomes this practical problem and adequate psychometric properties are reported. However, there are serious conceptual and clinical concerns about the diagnosis of BDDE (Carr, 2002), which question the focus and item content of this scale.

The Multi-Dimensional Body Self-Relations Questionnaire (MBSRQ; Brown, Cash, \& Mikulka, 1990) is a general body-image disturbance measure comprising 11 short scales including measures of cognitive-behavioural investment in appearance, appearance evaluation, and appearance satisfaction. In that the MBSRQ includes a number of scales not concerned with appearance per se, such as attitudes to health and fitness, it is a relatively insensitive measure of body-image disturbance. Also, the items do not sample the concerns of clinical populations and the important dimensions of social and behavioural dysfunction. Although it is a widely used scale (Sarwer, Wadden, \& Foster, 1998) it is perhaps most useful for its subscales, such as the Body Areas Satisfaction Scale, but more work is needed to establish the psychometric properties of these subscales.

Although there is a plethora of scales for assessing various aspects of body-image, and body-image disturbance (Cash \& Pruzinsky, 2002; Thompson et al., 1999), the field has been dogged by poor psychometric development and standardization, and a dearth of normative data. To address this problem, a well-standardized, psychometrically robust, factorial scale was developed - the Derriford Appearance Scale (DAS59; Carr et al., 2000). This scale is standardized on large clinical $(1,740)$ and non-clinical populations $(1,001)$ and measures the distress and dysfunction arising from body-image disturbance. Factor-analytic studies revealed a primary, general factor of self-consciousness of appearance, and a factorial structure reflecting particular problems in certain areas of functioning or with specific areas of the body, such as the face. Although the psychometric refinement and factorial structure of the DAS59 make it ideally suited to the detailed clinical investigation or assessment of the individual and to structured empirical enquiry, its length (59 items) may be less well suited to the more routine aggregation of data in clinical practice, to clinical audit, and to measurement where time is severely restricted.

Reconstructive plastic surgery for disfigurement arising from trauma, illness, and congenital factors is available in all National Health Service (NHS) regions. Concerns about appearance are widespread in the general population, and these are associated with adverse psychological effects of clinical significance in up to $7 \%$ of men and $15 \%$ of women (Harris \& Carr, 2001). The availability of a user-friendly, brief, and psychometrically sound scale to assess patient status and therapeutic effectiveness on a routine basis, offers the promise of rapidly building a large database that will facilitate necessary developments in therapeutic approaches.

This paper reports the development of a short form of the DAS24, which retains the psychometric robustness and conceptual identity of the long form and which provides the necessary brevity and ease of use as identified above. 


\section{Method}

\section{Overview}

Data were collected in a UK multi-centre trial from out-patients, and from patients on waiting lists for reconstructive plastic surgery. A Patient Survey Booklet (PSB) containing the DAS24 and the DAS59, and a range of appropriate psychometric scales was completed by each participant, either in the clinic (out-patients) or at home and returned by post (waiting list patients).

Data are also reported from a study of the general population, the details of which are reported elsewhere (Carr et al., 2000; Harris \& Carr, 2001).

\section{Materials}

The PSB comprised the seven tests described below and an initial instruction page, which also served to collect demographic information. The instructions also reiterated the ethical points included in the covering letter regarding the right to withdraw, patient anonymity, and directed the respondent to the authors' address and telephone number should further support be needed.

\section{Derriford Appearance Scale short form, DAS24}

The DAS24 was the measure under test. An introductory section enabled respondents to identify and describe the aspect of appearance about which they are most sensitive or self-conscious and is referred to as their 'feature' in scale items. Participants responses related to concerns and behaviours in relation to this feature within the context of overall body self-consciousness. Twenty-five items were selected on the basis of their psychometric properties, such as factor loadings, item-whole correlations and factor sampling, as well as their clinical utility, as established in trials of the DAS59 (Carr et al., 2000). Acceptable face-validity, as assessed in a pilot study of 45 plastic surgery outpatients (Moss, 1997a), was a further inclusion criterion for the items selected.

\section{Derriford Appearance Scale 59, DAS59}

The long form of the DAS59 (Carr et al., 2000) was included as the criterion measure to assess concurrent validity. The DAS59 is the only well-developed generic measure of distress and dysfunction in problems of appearance. It has high internal consistency $(\alpha=.98)$ and good test-retest reliability $(0.75$ for general population and 0.86 for patients). Good criterion and construct validity has also been demonstrated in psychometric and plastic surgery intervention trials.

Crown crisp experiential inventory (CCEI)

The CCEI is a general mental health screening measure. It has six subscales; free-floating anxiety, phobic anxiety, obsessionality, somatic anxiety, depression, and hysteria. Testretest reliability has been clearly demonstrated and is reported in the test manual at over 0.68 for all six subscales. Criterion validity with clinical observation is also adequate (Crown \& Crisp, 1979). The CCEI was included to allow the assessment of criterion validity. We predicted correlations of moderate effect size for all of the subscales with the exception of hysteria, which itself is used to test discriminant validity. 
Fear of negative evaluation (FNE)

The FNE measure is one aspect of social anxiety, as measured by Watson and Friend (1969). FNE was chosen as it appears close to some aspects of the self-reported problems of plastic and reconstructive surgery patients. It is defined as 'apprehension about others' evaluations, distress over their negative evaluations, avoidance of evaluative situations, and the expectation that others would evaluate oneself negatively' (p. 449). Test-retest reliability was reported by Watson and Friend as 0.78 . Internal reliability $(\alpha)$ was .72 . Criterion validity was acceptable when the scale was assessed in experimental situations.

Social avoidance and distress (SAD)

The SAD measure is the other aspect of social anxiety, as measured by Watson and Friend (1969). SAD is defined as a combination of 'avoiding being with, talking to, or escaping from others for any reason', and 'negative emotion, such as being upset, distressed, tense, or anxious, in social interaction, or the reported lack of positive emotion, such as being relaxed, calm, at ease, or comfortable' (p. 449). Test-retest reliability was reported by Watson and Friend as 0.68 . Internal reliability $(\alpha)$ was .77 . Criterion validity was acceptable when the scale was assessed in experimental situations.

An additional reason for choosing the Watson and Friend measures of social anxiety was the absence of items relating to physical appearance, thus avoiding spuriously high correlations with the DAS24.

\section{Positive affect negative affect schedule (PANAS)}

The PANAS was selected as a mood scale which separately measures positive and negative affect. Inter-correlation of the two scales has been reported by the authors Watson, Clark, and Tellegen (1988) as -.17 , implying orthogonality for all practical purposes. The design of the scale is such that it can be used as a trait or state scale, by altering the wording of the initial question. For the purposes of this study, it was used as a trait scale, requiring respondents to answer how they generally felt.

Test-retest reliability was reported as 0.68 for positive affect (PA), and 0.71 for negative affect (NA). Validity correlations with a number of other positive and negative affect scales produces correlations of .50-.89 for PA, and .51-.94 for NA.

\section{Internalized Shame Scale}

The affect of shame is highly relevant to the phenomenology of living with a different appearance. Gilbert, Pehl, and Allen (1994) describe the following experiences as characteristic of shame: '[Self seen as] object of scorn, disgust, ridicule, humiliation; paralysed, helpless, passive, inhibited; inferior, smaller, weaker; [subject to] involuntary body responses; rage, blush, tears, gaze avoidance; functioning poorly, mind going blank, desire to hide, conceal; self in focal awareness' (p. 26). The phenomenology of self-consciousness of appearance, although not identical, clearly overlaps that of shame as defined by Gilbert et al. (1994). Concerns about being negatively evaluated by others, the affective responses of humiliation and embarrassment, the concealment of facial and bodily features about which the person is sensitive, and the tendency to social avoidance are all characteristic of self-consciousness of appearance (Harris, 1982; Moss, 1997a, 1997b). Also, more recent development and elaboration of the concept of 
body-shame (Gilbert, 2002) shows close similarity to the concept of self-consciousness of appearance (Carr, 2002; Harris, 1982).

The Internalized Shame Scale was designed to measure trait shame. Cook (1994) reports high internal reliability (.95) and reasonable test-retest reliability (0.69). Various correlations with other measures are reported by Cook, associating aspects of shame with poor self-esteem (r's range from -0.52 to -0.77 ).

\section{Procedure}

With the assistance of the British Association of Aesthetic Plastic Surgeons (BAAPS), UK consultant plastic surgeons working in NHS units were invited by letter (DH) to participate in the study. Twenty-five surgeons from 13 units distributed throughout England, Wales, and Scotland agreed to participate by providing access to suitable patients. Ethical approval was obtained initially from Plymouth Hospital's NHS Trust and, subsequently, from other Trusts with participating units as appropriate.

\section{Waiting list patients}

A target sample of adult patients awaiting treatment for an objectively identifiable 'abnormality' of appearance and characterized by a range of body sites and aetiologies was identified. As men are underrepresented in this patient population, an attempt was made to ensure that a sufficiently large number of men was sampled to allow meaningful multivariate analysis. An even distribution across the age range 18-75 years was also attempted where this did not detract from the other criteria. No patients who were psychotic, who had dementia, or who were judged by their consultant to be too emotionally vulnerable to participate, were selected.

All patients were sent a PSB with a freepost envelope for its return when completed and a standard covering letter from their own consultant surgeon. This letter included a rationale for the patient's involvement and for the study in general, as well as meeting the ethical requirements for informed consent and right to withdraw. The letter also included the telephone number of the research office as a contact point for participants. After 1 month, patients who had not returned their booklets were again contacted by letter and requested to do so.

\section{Out-patients}

Participating surgeons identified patients using the inclusion and exclusion criteria described above. Patients who agreed to take part in the study were given PSBs to complete following their outpatient appointments. All booklets were returned to the central research office in Plymouth by identified clinic staff within each collaborating institution.

\section{Ethical issues}

All patients received the necessary information to give informed consent. Covering letters (waiting list) or information sheets (out-patients) clarified the right to withdraw and the anonymity of patients' data. For waiting list patients, contact information was provided and clinical support was available within the team (TC) by referral. The concern for well-being of out-patients was covered within their clinical services. 


\section{Participants}

From the out-patient clinic route, 271 usable booklets (approximately 80\%) were returned. To recruit patients through the waiting lists, 680 patients were contacted. Of these, 264 returned usable booklets within 4 weeks, or after a single prompt. Respondents were included if they had correctly completed at least the DAS24. The total sample size is therefore 535. A further 93 waiting list patients returned unusable booklets, thus demonstrating an acceptable 52\% response rate. A sample of nonrespondents $(N=46)$ chosen at random and followed-up by telephone demonstrated that perceived irrelevance was the most prominent reason for non-return of the booklet. Any potential bias related to the response rate is thus one of over-representation of clinical need. However, comparison with earlier large studies in this area (Carr et al., 2000) and between the two sampling methods (out-patient and waiting lists) does not indicate a significant response bias. No participants reported distress upon receipt of the booklet.

The sample population included 147 (27.5\%) men, and 388 (72.5\%) women. The sample was examined in terms of the age of the respondents. A wide range of ages between 18 and 90 was included in the sample, with a skew towards the age band of 20 to 40 years old and a full and representative range of feature sites (Table 1). The range of disfigurement aetiology was also fully represented; congenital malformation (e.g. cleft lip, haemangiomas), scarring from trauma and burns, disease (e.g. skin cancer, acne), developmental growth (e.g. breasts, nose), obesity and weight loss, effects of pregnancy and breast feeding, and facial ageing.

Table I. Distributions of age bands and body sites with mean DAS24 scores (Totals are less than 535 due to incomplete data for age and/or body site among some respondents)

\begin{tabular}{lcclcc}
\hline Age & $N(\%)$ & DAS(24) mean & Body site & $N(\%)$ & DAS(24) mean \\
\hline $18-19$ & $22(4)$ & 42.4 & Trunk and genitalia & $200(38)$ & 55.5 \\
$20-29$ & $167(32)$ & 49.4 & Lower limb & $54(10)$ & 48.1 \\
$30-39$ & $149(29)$ & 52.0 & Upper limb & $43(8)$ & 43.2 \\
$40-49$ & $99(19)$ & 48.3 & Face & $182(34)$ & 43.3 \\
$50-59$ & $41(8)$ & 41.0 & Head and neck & $49(9)$ & 38.0 \\
$60-69$ & $26(5)$ & 32.2 & (excluding face) & \\
$70+$ & $14(3)$ & 33.5 & & & \\
Total & $518(100)$ & & Total & $528(99)$ & \\
\hline
\end{tabular}

\section{Results}

\section{Impact of data collection method on DAS24 total scores}

The two data collection methods, through out-patient clinics and from waiting lists, were contrasted to determine whether any selection bias had systematically influenced the results. The means and standard deviations of total scores were highly similar (outpatients, $M=47.2, S D=18.4$; waiting list patients $M=48.2, S D=17.3$ ) and not significantly different $(F=0.46 ; d f=1,532 ; p=.496)$.

\section{Item analyses}

Means and standard deviations of all items were examined. With the exception of one item, 'I avoid getting my hair wet', with a very low mean and standard deviation 
$(M=1.4, S D=0.88)$, all items demonstrated acceptable degrees of skew and kurtosis, full use of the response categories for each item, and mean scores around the centre of the distribution.

The second stage of the item analysis was to examine the item-total correlation. A strong item-total correlation reflects good discrimination, providing that the scale as a whole is discriminating, and that the item contributes strongly to the scale total. Corrected item-total correlations ranged from 46 to 72 (Table 2), again with the exception of the 'wet hair' item, which correlated at only .06. Cronbach's alpha for the scale was .92, indicating good internal reliability, which was not changed to any significant degree by the exclusion of any one item.

At this stage, due to the poor performance of the 'wet hair' item, it was omitted from the scale and from all subsequent analyses. The final scale thus comprised 24 items and this number identifies the short form of the DAS24.

Table 2. Item-total correlations for Derriford Appearance Scale short form (DAS24) corrected for reverse scoring of items where relevant

\begin{tabular}{llc}
\hline Item number & Item summary & $\begin{array}{c}\text { Corrected item-total } \\
\text { correlation (Pearson's r) }\end{array}$ \\
\hline 1 & Feeling confident & 0.5 I \\
2 & Distress at reflection & 0.72 \\
3 & Irritable at home & 0.72 \\
4 & Feel hurt & 0.67 \\
5 & Self consciousness affects work & 0.46 \\
6 & Distressed at beach & 0.60 \\
7 & Misjudged due to appearance & 0.50 \\
8 & Feel feminine/masculine & 0.57 \\
9 & Self conscious of appearance & 0.67 \\
10 & Feel irritable & 0.63 \\
11 & Adopt concealing gestures & 0.56 \\
12 & Avoid communal changing & 0.60 \\
13 & Distressed in supermarkets/dept. stores & 0.50 \\
14 & Feel rejected & 0.66 \\
15 & Avoid undressing with partner & 0.58 \\
16 & Distressed playing sport/games & 0.53 \\
17 & Close into shell & 0.63 \\
18 & Distressed by clothing limitations & 0.64 \\
19 & Distressed at social events & 0.68 \\
20 & Feel normal & 0.64 \\
21 & Affects sex life & 0.65 \\
22 & Avoid leaving house & 0.48 \\
23 & Distressed at others remarks about appearance & 0.61 \\
24 & Avoid pubs/restaurants & 0.53 \\
\hline
\end{tabular}

\section{Psychometric properties of the DAS24}

Test-retest reliability

The waiting list sample was targetted for test-retest reliability after 6 months. Of the 264 potential participants, 136 (52\%) replies were received. Seventy patients were now post-operative, and thus inappropriate for inclusion in test-retest data. Eight responses 
indicated the participant was no longer contactable at the address. Sixty-six participants thus provided test-retest data. The test-retest correlation was 0.82 . This is entirely comparible with the figure from the general population study $(r=.88)$, which was obtained over an interval of 3 months (Harris \& Carr, 2001).

The distribution of total scores was very close to normal (see Fig. 1), showing a slight positive skew and 'flattened' distribution. This flattening is beneficial when designing a scale to discriminate amongst a population (as is the intention of this scale), as those respondents in the second and third quartiles are more evenly spread, rather than bunching around the mean. The distribution of total scores is shown in Fig. 1.

Validity of DAS24

Concurrent validity was determined by comparison with the criterion measure, the DAS59. A Pearson correlation of $.88(p<.0001, d f=533)$ demonstrates the excellent relationship between the two measures.

The convergent and discriminant construct validity of the DAS24 was assessed by Pearson correlations with other scales chosen a priori.

It was hypothesized that there would be good (.5-.7) positive correlations with the CCEI total, the CCEI anxiety subscale, the CCEI depression subscale (the two factors of social anxiety measured by Watson \& Friend, 1969), social avoidance and distress, and fear of negative evaluation, negative affect (measured by the PANAS) and shame (measured by the Internalized Shame Scale). Moderate positive correlations were found. Discriminant validity was tested by anticipated low correlation with CCEI hysteria, and negative correlation with positive affect, measured by the PANAS. The low correlation with hysteria $(r=.08)$ was as anticipated, as was the direction of the correlation with positive affect, but the positive affect correlation was smaller than expected. These findings are summarized in Table 3.

\section{Demographic characteristics: Clinical population}

Age

Where age was known, we observed a small but significant negative correlation with the DAS24 (p. 9; $r=-.20, N=518, p<.01$ ). This indicated some tendency for better adjustment to problems of appearance with greater age, in line with intuitive expectation. Mean scores for age bandings are shown in Table 1.

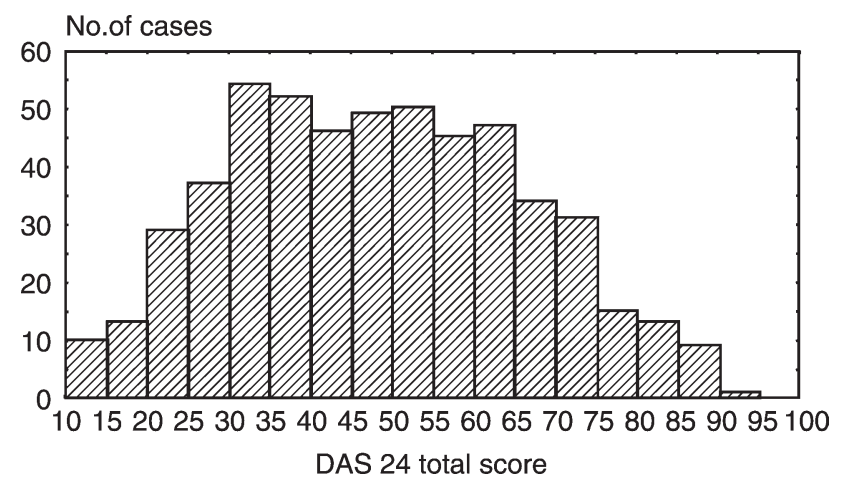

Figure I. Frequency distribution of DAS24 scores of 535 clinical respondents. 
Table 3. Convergent and discriminant construct validity of DAS24

\begin{tabular}{lc}
\hline Measure & Correlation with DAS24 (Pearson's $r$ ) \\
\hline CCEI total & 0.51 \\
CCEI anxiety & 0.50 \\
CCEI depression & 0.45 \\
Social avoidance and distress & 0.53 \\
Fear of negative evaluation & 0.50 \\
Negative affect & 0.50 \\
Shame & 0.66 \\
CCEI hysteria & 0.08 \\
Positive affect & -0.24 \\
\hline
\end{tabular}

$N=535$.

\section{Gender}

Women had a mean score of $50.75(S D=17.2)$ and men a mean of $39.62(S D=17.1)$. A one-way ANOVA showed that this difference was highly significant $(F=44.7$; $d f=1,532 ; p<.0001)$

\section{Body sites}

There was a clear association between body sites and the mean scores of participants reporting concern with features at those body sites. The highest scores were associated with concern about the trunk (including breasts) and the lower limbs. Lowest scores were associated with the head (excluding the face). A one-way ANOVA showed a significant effect of body site $(F=18.4 ; d f=4,523 ; p<.0001)$. The mean scores for body sites are shown in Table 1.

\section{General population data}

In a separate study reported in detail elsewhere (Harris \& Carr, 2001), copies of the DAS24 were mailed to a representative general population sample $(N=2,700)$ selected randomly with constraints for age (18 years and over), gender and socio-economic status based on postcodes. There were 1,107 useable responses extracted from the 1,302 replies ( $48 \%$ target population) as shown in Table 4

Of the 1,107 respondents, 51\% reported a concern about appearance and $71 \%$ of these were female. The bulk of the concerns related to the face (41\%) and the mean DAS24 score for those concerned about appearance (39.4\%) was significantly higher than those not concerned about their appearance (22.3\%), $t=26.29$, $d f=895$, $p<.001$.

The mean scores for the total general population and the clinical population are significantly different $(t=19.03, d f=11,537, p<.0005$; two-tailed, independent samples, accounting for different sample variances).

The mean DAS24 scores for two age bands in the general population and for facial and sexual bodily features, for males and females are shown in Table 5.

For females and males, mean scores are higher at younger ages and female scores are significantly higher than male scores overall $(t=3.76, d f=329, p<.001)$. 
Table 4. General population sample $(N=1,107)$

\begin{tabular}{lccc}
\hline & $\begin{array}{c}\text { Concerned by } \\
\text { appearance }\end{array}$ & $\begin{array}{c}\text { Not concerned by } \\
\text { appearance }\end{array}$ & $\begin{array}{c}\text { Total general } \\
\text { population }\end{array}$ \\
\hline Number & 564 & 543 & 1,107 \\
Male & $166(29 \%)$ & $323(59 \%)$ & $489(44 \%)$ \\
$\quad$ Mean age (SD) & $42.9(17.1)$ & $49.8(17.5)$ & $47.5(17.6)$ \\
Female & $398(71 \%)$ & $220(41 \%)$ & $618(56 \%)$ \\
$\quad$ Mean age (SD) & $40.7(14.7)$ & $48.8(17.2)$ & $43.6(16.1)$ \\
Features of concern & $135(24 \%)$ & $0(0 \%)$ & $135(12 \%)$ \\
$\quad$ Sexual & & $0(0 \%)$ & $232(21 \%)$ \\
Facial $^{\text {b }}$ & $232(41 \%)$ & $0(0 \%)$ & $197(18 \%)$ \\
Other & $197(35 \%)$ & $543(100 \%)$ & $543(49 \%)$ \\
$\quad$ None & $0(0 \%)$ & $22.28(7.55)$ & $30.99(13.88)$ \\
DAS24 score mean (SD) & $39.39(13.40)$ & &
\end{tabular}

${ }^{a}$ Breasts/chest, abdomen, buttocks, hips and thighs.

${ }^{\mathrm{b}}$ All features from the neck up (inclusive).

Table 5. Mean (SD) DAS24 scores for the general population

\begin{tabular}{|c|c|c|c|c|}
\hline & \multicolumn{2}{|l|}{ Males } & \multicolumn{2}{|l|}{ Females } \\
\hline & DAS24 score Mean (SD) & $N$ & DAS24 score Mean (SD) & $N$ \\
\hline All ages & $26.87(11.57)$ & 489 & $34.27(14.67)$ & 618 \\
\hline $18-49$ years & $29.04(12.10)$ & 256 & $37.09(15.13)$ & 385 \\
\hline 50 years and over & $24.48(10.47)$ & 233 & $26.06(12.59)$ & 233 \\
\hline \multicolumn{5}{|c|}{ Concerned about appearance } \\
\hline Facial features ${ }^{\mathrm{a}}$ & $33.69(1 \mathrm{I} .5 \mathrm{I})$ & 82 & $36.40(11.52)$ & 150 \\
\hline Sexual features ${ }^{b}$ & $38.32(9.62)$ & 25 & $43.77(\mid 4.7 I)$ & 110 \\
\hline Other & $38.39(14.95)$ & 59 & $43.15(13.14)$ & 138 \\
\hline Not concerned & $22.14(7.32)$ & 323 & $22.49(7.91)$ & 220 \\
\hline
\end{tabular}

${ }^{\text {a } B r e a s t s / c h e s t, ~ a b d o m e n, ~ b u t t o c k s, ~ h i p s ~ a n d ~ t h i g h s . ~}$

${ }^{\mathrm{b}}$ All features from the neck up (inclusive).

Test-retest reliability

Of the respondents from the general population, 195 (18\%) provided retest data after an interval of 3 months. The retest reliability coefficient was 0.88 which compares well with that obtained from the clinical population after an interval of 6 months $(0.82)$.

\section{Discussion}

This study describes the development of a psychometrically robust short form of the original DAS24 (Carr et al., 2000) that has the advantage of brevity while maintaining a known and strong relationship with the original factorial scale. The correlation between the scale totals (0.88) demonstrates the necessary strong relationship, and, together with the good test-retest reliabilities (0.82, clinical population; 0.88 , general population), indicates that the DAS24 may be used with confidence in applications where there is more time pressure and when the detail provided by the factorial structure of the DAS59 is not required. 
The item analyses and score distribution (see Table 2 and Fig. 1) show that all items contribute strongly to the scale total and provide a wide distribution of scores that allows effective discrimination between individuals and groups. The high level of internal consistency (Cronbach $\alpha=.92)$ and the good item-total correlations $(92 \%$ at $r=.5$ or better) suggest that the item sampling from the original scale effectively represented the item set and that the total score provided by these 24 items reflects the main factor of self-consciousness of appearance underlying the original scale (Carr et al., 2000).

Good construct validity of the DAS24 is demonstrated in (1) the predicted convergent and discriminant correlations with other established scales (Table 3); (2) the age distribution of scores, showing the maximum frequency of, and concern about, appearance problems occurring between the ages of 20 and 39 years of age, falling to a minimum after 70 years of age (Table 1); and (3) the higher scores for patient groups compared with the general population (Tables 1, 4, and 5), and for general population samples reporting concern about appearance compared with those reporting no such concern (Table 4).

Good content and face validities have been achieved by developing items originally from patients' reports of their difficulties (Harris, 1982). The scale covers the full range of distress and dysfunction associated with problems of appearance from patients whose lives are dominated by their body-image disturbance and self-consciousness of appearance to those for whom appearance is of no concern. It is highly acceptable to people with a problem of appearance and to those with none, to people whose problem of appearance is visible to others and those whose problem of appearance is apparent only to themselves.

It seems likely that the scale's introductory section, through which the features of concern for each responding individual are identified and the provision of a 'not applicable' response category throughout, has created the flexibility that allows people with and without problems of appearance and pre- and post-operative patients to use the scale without difficulty. However, we should note that the standardization samples did not include people who had overcome major problems of appearance without surgical intervention, either through their own unsupported efforts or with psychotherapeutic assistance. It is possible that the problem-oriented nature of many items could be less acceptable to this group and this will be clarified only through future research. It was our intention to develop a short scale to reliably and validly assess the dysfunction and distress arising from problems of appearance, and this has been achieved.

Table 1 shows that the degree of distress and dysfunction arising from a problem of appearance is not determined by its visibility. This confirms the findings previously obtained with the long form of the DAS59 scale (Carr et al., 2000). The independence of dysfunction and distress from the degree of visible disfigurement has been observed by a number of authors (e.g. Moss, 1997b) and highlights the need for valid and reliable assessment. The general importance given to appearance is demonstrated by the widespread occurrence of concerns about appearance in the general population sample (Table 4). There can be little doubt that the current media promotion of an ideal of youth and beauty contributes to contemporary levels of unease about appearance (Rumsey, 1997; Gilbert \& Miles, 2002) but the normality of some concern about appearance places the concerns of patients on a continuum with the population at large. This continuity between clinical and 'normal' concerns about appearance poses fundamental questions for contemporary theorists (e.g. Kent \& Thompson, 2002). 
There is much work to be done on the aetiology of self-consciousness of appearance and body-image disturbance and many conditions that are largely unexplored. The DAS24 will be highly useful as an instrument with which to screen for selfconsciousness of appearance in a broad range of patient groups whose medical and surgical conditions, while not primarily appearance related, may cause disfigurement, deformity or other problems of appearance, for example, eczema, psoriasis, neurofibromatosis, mastectomy, scoliosis, amputation, thyroid eye disease, and colostomy (Harris, 1997).

In primary medical care, the DAS24 will help assess the clinical needs of patients who request referral for cosmetic surgery, and if used sensitively alongside clinical judgments of health professionals, and bearing in mind the possibility of potentially exaggerated claims of need, the scale will inform decision-making when prioritizing resource allocation for treatment of appearance related problems under the NHS. In everyday clinical practice, the DAS24 can also provide plastic and reconstructive surgeons with reliable and valid data to guide clinical decision-making and to support audit and clinical governance procedures.

The further development of more effective physical and psychological interventions for people suffering with disfigurements, body-image disturbance, and self-consciousness of appearance depends upon the availability of well standardized, valid and reliable scales of measurement to support the necessary research. The DAS24 is a short, userfriendly scale with robust psychometric characteristics that is ideally suited to outcome studies and to the routine aggregation of data in a wide variety of settings.

\section{References}

Brown, T. A., Cash, T. F., \& Mikulka, P. J. (1990). Attitudinal body-image assessment: Factor analysis of the body self-relations questionnaire. Journal of Personality Assessment, 55, 135-144.

Carr, A. T. (1997a). Assessment and measurement in clinical practice. In R. Lansdown, N. Rumsey, E. Bradbury, T. Carr, \& J. Partridge (Eds.), Visibly different: Coping with disfigurement (pp. 131-146). Oxford: Butterworth-Heinemann.

Carr, A. T. (1997b). Physical and psychological therapies in disfigurement: A selective overview. Paper presented to the Annual Conference of the Disfigurement Interest Group, King's Fund, London.

Carr, A. T. (2002). Body shame: Issues of measurement and assessment. In P. Gilbert \& J. Miles (Eds.), Body shame: Conceptualisation, research and treatment (pp.90-102). Hove: Brunner-Routledge.

Carr, A. T., Harris, D. L., \& James, C. (2000). The Derriford Appearance Scale: A new scale to measure individual responses to living with problems of appearance. British Journal of Health Psychology, 5, 201-215.

Cash, T. F., \& Labarge, A. S. (1996). Development of the appearance schemas inventory: A new cognitive body-image assessment. Cognitive Therapy and Research, 20, 37-50.

Cash, T. F., \& Pruzinsky, T. (2002). Body Image: A bandbook of theory, research and clinical practice. New York, New York: Guilford.

Cook, D. R. (1994). Internalized Shame Scale: Professional manual. Menomonie, W1: Channel Press, Available from author: E 5886 803rd Ave., Menomonie, W1 54751.

Cooper, Z., \& Fairburn, C. G. (1987). The eating disorder examination: A semi-structured interview for the assessment of the specific psychopathology of eating disorders. International Journal of Eating Disorders, 6, 1-8.

Crown, S., \& Crisp, A. H. (1979). Manual of the Crown Crisp Experiential Index. London: Hodder and Stoughton. 
Gilbert, P. (2002). Body shame: A biopsychosocial conceptualisation and overview with treatment implications. In P. Gilbert \& J. Miles (Eds.), Body shame: Conceptualisation, research and treatment. Hove: Brunner-Routledge.

Gilbert, P., and Miles, J. (2002). Body shame: Conceptualisation, research and treatment. Hove: Brunner-Routledge.

Gilbert, P., Pehl, J., \& Allan, S. (1994). The phenomenology of shame and guilt: An empirical investigation. British Journal of Medical Psychology, 67, 23-36.

Grant, J., \& Cash, T. F. (1995). Cognitive behavioral body-image therapy: Comparative efficacy of group and modest-contact treatments. Behavior Therapy, 26, 69-84.

Harris, D. L. (1982). The symptomatology of abnormal appearance: An anecdotal survey. British Journal of Plastic Surgery, 35, 312-323.

Harris, D. L. (1997). Types, causes and physical treatment of visible differences. In R. Lansdown, N. Rumsey, E. Bradbury, T. Carr, \& J. Partridge (Eds.), Visibly different: Coping with disfigurement (pp. 79-90). Oxford: Butterworth-Heinemann.

Harris, D. L., \& Carr, A. T. (2001). Prevalence of concern about physical appearance in the general population. British Journal of Plastic Surgery, 54, 223-226.

Kent, G., \& Thompson, A. R. (2002). The development and maintenance of shame in disfigurement: Implications for treatment. In P. Gilbert \& T. Miles (Eds.), Body shame: Conceptualisation, research and treatment (pp. 103-116). Hove: Brunner-Routledge.

Labarge, A. S., Cash, T. F., \& Brown, T. A. (1998). Use of a modified stroop task to examine appearance schematic information processing in college women. Cognitive Therapy and Research, 22, 179-190.

Moss, T. P. (1997a). Individual differences in adjustment to perceived abnormalities of appearance. Unpublished $\mathrm{PhD}$ Thesis, University of Plymouth.

Moss, T. P. (1997b). Individual variation in adjustment to visible differences. In R. Lansdown, N. Rumsey, E. Bradbury, T. Carr, \& J. Partridge (Eds.), Visibly different: Coping with disfigurement (pp. 121-130). Oxford: Butterworth-Heinemann.

Rosen, J. C., \& Ramirez, E. (1998). A comparison of eating disorders and body dysmorphic disorder on body image and psychological adjustment. Journal of Psychosomatic Research, 44, 441-449.

Rosen, J. C., Reiter, J., \& Orosan, P. (1995). Assessment of body image in eating disorders with the body dysmorphic disorder examination. Behaviour Research and Therapy, 33, 77-84.

Rosen, J. C., Srebnik, D., Saltzburg, E., \& Wendt, S. (1991). Development of a body image avoidance questionnaire. Psychological Assessment: Journal of Consulting and Clinical Psychology, 3, 32-37.

Rumsey, N. (1997). Historical and anthropological perspectives on appearance. In R. Lansdown, N. Rumsey, E. Bradbury, T. Carr, \& J. Partridge (Eds.), Visibly different: Coping with disfigurement (pp. 91-101). Oxford: Butterworth-Heinemann.

Sarwer, D. B., Wadden, T. A., \& Foster, G. D. (1998). Assessment of body image dissatisfaction in obese women: Specificity, severity and clinical significance. Journal of Consulting and Clinical Psychology, 66, 651-665.

Slade, P. D., \& Russell, G. F. M. (1973). Awareness of body dimensions in anorexia nervosa: Crosssectional and longitudinal studies. Psychological Medicine, 3, 188-199.

Thompson, J. K., Heinberg, L. J., Altabe, M., \& Tantleff-Dunn, S. (1999). Exacting beauty: Theory, assessment and treatment of body-image disturbance. Washington DC: American Psychological Association.

Watson, D., Clark, L. A., \& Tellegen, A. (1988). Development and validation of brief measures of positive and negative affect: The PANAS Scales. Journal of Personality and Social Psychology, 54(6), 1063-1070.

Watson, D., \& Friend, R. (1969). Measurement of social evaluative anxiety. Journal of Consulting and Clinical Psychology, 33, 448-457.

Received 4 February 2003; revised version received 19 March 2004 